\title{
A Profit Maximization Scheme for Enhancing Quality of Service (QoS) in Cloud Computing
}

\author{
Poonam P. Khot \\ ME Department of Computer \\ engineering JSCOE Hadapsar, \\ pune, India.
}

\author{
S. D. Satav \\ ME Department of Information Technology, \\ JSCOE Hadapsar, \\ pune,India.
}

\begin{abstract}
Internet basis computing that is depending upon on-demand work is called as Cloud computing. Cloud computing provides shared resources as well as data over the network to user on the basis of his demand. Cloud computing has very essential part that is called as the cloud economics analysis. In cloud economics, work of enhancement of profit is accomplished. Large benefit is the most essential aspect as correspondence with cloud service providers and also depending upon markets demand through the management of cloud service platform it is compelled. Initially, determination of the cost as well as revenue for increasing the profit is very essential. The user satisfaction in profit increment is additionally considered as the cost of the cloud. Under the cost, both the renting cost as well as energy utilization cost also considered. To increase the profit there is must decrease the cost. To minimize the cost have to configure the server accurately. At the time of server configuration, computing is done over the assumed waiting time as well as service charge. Existing cloud providers was utilized a single long-term strategy to setup cloud platform. But this single long-term renting strategy has the issue of unable to provide the service with the high quality and additionally leads wasting the resources. To solve this issue, a system called Double resource Renting (RR) is developed. This concept includes the both short term as well as long-term renting methodologies. Double resource renting methodology ensures the quality of service and minimizes the wastage of resources. Previous system also implemented double renting system, but only for the homogeneous cloud scenario. By comparing both heterogeneous as well as homogeneous environment, the study says that a heterogeneous environment is most difficult. So, to solve this drawback, proposed system is working over a heterogeneous environment.
\end{abstract}

\section{Keywords}

Cloud Service Providers, Double Renting Scheme, Profit Maximization, Single Renting Scheme.

\section{INTRODUCTION}

The cloud is an advanced stage that gives high accessibility, dynamic resource pools, and virtualization. Presently a days, cloud computing implies being able to use versatile, distributed computing situations inside the limits of the Internet. The most ideal route for the conveyance of facilitated services over is called as Internet Cloud computing. The cloud computing gives a non-useful and undemanding answer for continuous computing. The focal point of Prevailing cloud frameworks are on recognizing a successful solution for the overseeing different resources. Cloud computing in that virtual shared servers give software, infrastructure, platform, devices as resources to clients on a premise of pay-as-you utilize. To get to your data from any place whenever is conceivable by utilizing cloud. Cloud computing opens a path for a client what you require and Pay for what you Use model. Through this it opens an entryway of organization to dedicate on novel one of kind solutions which will offers hands to comprehend key client problem.

Cloud computing is empower simple on interest network access to a mutual pool of configurable computing resources that will be quickly appoint and discharged with insignificant administration or without association with service supplier. Cloud explains the utilization of an set of services, software's, infrastructure and data contained pools of computer, network, data and storage resources. These segments can be scaled up or down, obliging an on interest utility like model of usage and designation. Cloud enhances facilitated exertion, availability, scaling as well as openness and provides the likelihood to diminishment of expense through productive and upgraded calculation. In business thoughts the advantage is the imperative component to be exist in the field of the particular environment. The requirement of benefit increment in cloud computing environment is needed. 60 billion servers are start now working in this world. So the server required a colossal energy. To save the utilization of power, requirement of greatest use of resources is critical. Guessing of burden, examination of burden, stability of various frameworks, framework execution, communication between the nodes, and way of work to be exchanged, choosing of nodes and numerous different ones is the significant problem to determine while making such algorithms. This load taken in record can be similarly as CPU burden, memory utilized and defer in burden. A dynamic load balancing plan should be proposed for preventing over-loaded and under-loaded situations in cloud framework. Hence the issue of dynamic Application arrangement will be tended to for allotting employments to machines taking into account their changing requests in heterogeneous cloud situations.

All things considered, a service supplier rents certain number of servers from the infrastructure suppliers and develops different multi-server structures for different application areas. Each multi-server system is to execute an exceptional type of service solicitations and applications. Along these lines, the renting expense is straight forwardly subject to the quantity of servers in a multi-server structure. The force use of a multi-server system is straight forwardly reliant to the quantity of servers and the server utilize, and to the square of execution pace. The salary of a service supplier is related to the way of service and the measure of service. To finish up, the advantage of a service supplier is generally managed by the arrangement of its service stage.

Some document storage services which are online charges space on premise of per-gigabyte, and may charge additional for a bandwidth cost segment. For the most part these will be charged month to month or yearly; A couple of associations offer the services for free, based upon advancing income. 
Some hosting services don't put any restriction over the measure of space the customer's record can utilize. Some services demons a download some software which creates records only open on PCs which have that software; others grant customers to retain files by any web browser. With the extended inbox space offered by webmail services, various customers have started using their webmail services as an online storage. A couple of areas offer free boundless record storage but have a size restriction over documents. A couple of websites offer additional online storage restriction consequently for new client referrals.

The increment of benefit is accomplished here. In this, service charge for every service that is demanded by the client is ascertained. Commonly between the customer and server has some understanding. In this service level understanding, Given the QoS requires to oblige the customer and the most needed execution time. If the service supplier harms this SLA no charge is suited the particular service. So there will be the loss of the advantage. Here calculation the perfect speed and size of the data the service level agreement is provided and here an assessing model is created by size and speed and service charge is calculated. So the service supplier can increase the advantage.

\section{PROBLEM STATEMENT}

Major thing is that the increasing the benefits in case of quality as well as price by implementing Double Quality Guaranteed technique. Existing system utilizes method depended on Single Quality Unguaranteed method which has minimum benefits in case of quality and price.

\section{LITERATURE SURVEY}

G. P. Cachon and P. Feldman [2] illustrates why an organization may support static assessing over component esteeming when clients are critical and pick whether to determine to purchase considering the organization is selected assessing framework. By charging a static value a organization powers an apportioning hazard on buyers however a organization that movements costs capably empowers a value risk on clients. The problem with component computing is that the organization may charge a high value that leaves purchasers with zero over abundance, so the organization will improve its earnings by realizing an assessing strategy that leaves clients with a positive surplus in all states of premium.

J. Cao, K. Hwang, K. Li, and A. Y. Zomaya [3] discussed the problem of ideal multi-server setup for maximizing profits in a cloud computing environment. The technique is to see a multi-server framework as a $\mathrm{M} / \mathrm{M} / \mathrm{m}$ queing model, like that upgrade problem can be characterized and appreciated diagnostically. They take two server speed and energy use models in record, to be particular, the unmoving pace model and the predictable rate model.

A. Fox, R. Griffith, A. Joseph, R. Katz, A. Konwinski, G. Lee, D. Patterson, A. Rabkin, and I. Stoica [4] illustrated cloud computing, the long-held long to process as an utility, can a clearing bit of the IT business, making software an incredible agreement all the more beguiling as an association and molding the way IT equipment is made and got. Engineers with innovative considerations for new Internet benefits circumstances no more need the broad capital expenses in equipment to send their association or the human cost to work it.

R. Buyya, C. S. Yeo, S. Venugopal, J. Broberg, and I. Brandic[5] disclosed Clouds are expected to offer services to external customers; supplier will be compensated for sharing their capabilities and resources. In this, authors have proposed plan for market-oriented assignment of resources within Clouds. The best in class Cloud methods have obliged support for market-oriented resource service and they will be extended to reinforce transaction of QoS between customers and supplier to set up SLAs frameworks and algorithm for dispersion of VM resources for meet SLAs; besides, threats worry with the breaking of SLAs.

P. Mell and T. Grance [6] illustrated advancing world view as a cloud computing. The NIST definition describes essential parts of cloud computing and provided to serve as methods for wide correlations of cloud services and implementation procedures, the implementation models and services characterized structure a basic scientific classification that is not proposed to endorse or compel a specific strategy for arrangement, service conveyance or business operation.

J. Chen, C. Wang, B. B. Zhou, L. Sun, Y. C. Lee, and A. Y. Zomaya [7] illustrated the utilization of utility theory got from financial aspects and for measuring consumer satisfaction inside cloud for producing another utility model. In light of the utility model, they plot a framework to support utilitybased SLAs with a particular end objective to conform the execution of employments and the cost of making them run. Determine an infrastructure as-a-service (such as Amazon EC2), where a business provider leases virtual machine case with spot costs from the cloud and picks up pay by giving service to its clients.

J. Mei, K. Li, J. Hu, S. Yin, and E. H.-M. Sha [8] talk about energy-aware scheduling decreases the energy use of CPU in hard ongoing systems through dynamic voltage scaling (DVS).The essential concept of energy-aware scheduling is to find slacks available to task and minimize CPU's frequency or further more cut down its voltage using the found slacks.

P. de Langen and B. Juurlink[9] depict exactly when top execution is futile, Dynamic Voltage Scaling (DVS) may use to diminish the dynamic power usage of embedded multi processors. In this, spillage aware arranging heuristics are shown that choose the best trade off from these three techniques: DVS, processor shutdown and finding the perfect number of processors. The results exhibit that the essentialness diminishment achieved by the best methodology is close to these speculative points of confinement.

S. Liu, S. Ren, G. Quan, M. Zhao, and S. Ren [10] determines a cloud service supplier working geologically conveyed data centers in a multi-power market environment and propose an essential efficient, advantage and cost-aware sales dispatching and resource task computation to extend an service supplier's net profit. Structure arranges the net profit boost problem as a constrained streamlining problem, using a bound together errand model getting diverse cloud layers.

Z. Liu, S. Wang, Q. Sun, H. Zou, and F. Yang [11] begins with develop a cloud service request model with SLA limitations and after that provides a cost-aware service request arranging path in case of genetic algorithm. By tweaked components of customer sales and the present structure stack, the approach can't simply lease and reuse virtual resources on enthusiasm to fulfill perfect arranging of dynamic cloud service requests in sensible time, however can likewise decrease the rental cost of the general base for extending SaaS provider an profits while meeting SLA requirements. 


\section{IMPLEMENTATION DETAILS}

\subsection{System Overview}

System consist of a novel renting technique for service providers. Proposed system satisfies quality of-service needs and also the system obtains more probable profit. Proposed novel double renting method that combines the long-term renting and short-term renting and this combination will provides quality-of-service requirements under the numerous system workloads as well as decreases wastage of the resources.

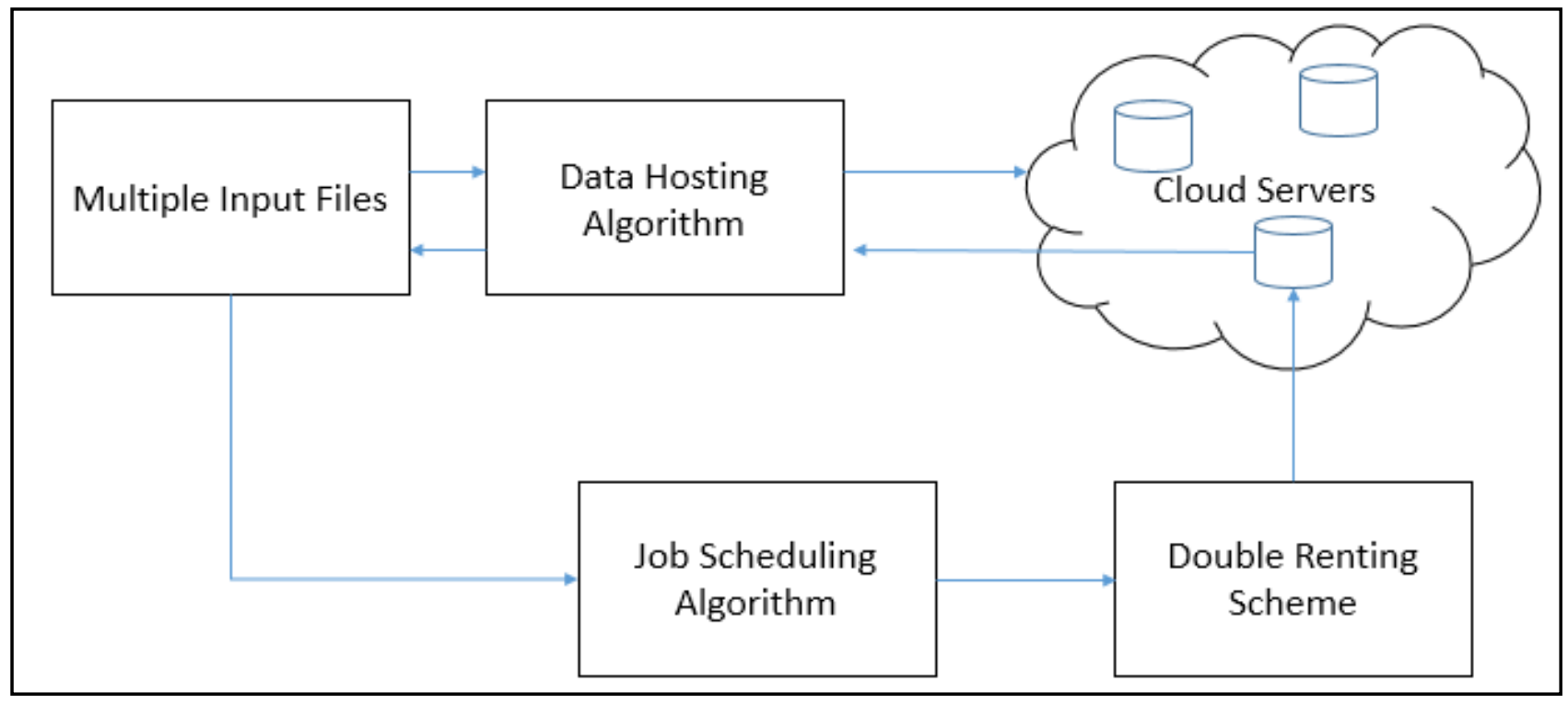

Fig 1: System Architecture

Implemented system is multi-server system and it is known as a $\mathrm{M} / \mathrm{M} / \mathrm{m}+\mathrm{D}$ queuing model. Performance of the system examined, such as the average service charge, the ratio of demands that require short term servers etc. The optimal arrangement problem of service provider's revenue driven enhancement is described as well as two types of conceptualized arrangements, i.e., the perfect arrangements and the actual solutions, are acquired individually. Steps of calculations are provided to assure the execution of technique. The proposed Double-Quality-Guaranteed (DQG) renting technique is more profitable as compared with the analyzed Single-Quality-Unguaranteed (SQU) renting technique in case of assuring the service quality. At the end this system is performed and observed over the heterogeneous cloud service provider platform.

\subsection{Mathematical Model}

$\mathrm{M}=(\mathrm{Q}, \Sigma, \mathrm{P}, \mathrm{q} 0, \mathrm{~F})$

Where

Q is the set of States

$\sum$ is the set of inputs

P State Transition Table

q0 is the initial Stage

$\mathrm{F}$ is the final Stage

$\mathrm{Q}=\left\{F_{m}, F_{s}, F_{o}\right\}$

Where $F_{m}=\left\{H_{r}\right\}$

Where $H_{r}$ : Heuristic Algorithm
$F_{s}=\{\mathrm{F} 1 ; \mathrm{F} 2 ; \mathrm{F} 3\}$

F1 : Calculate availability of selected cloud.

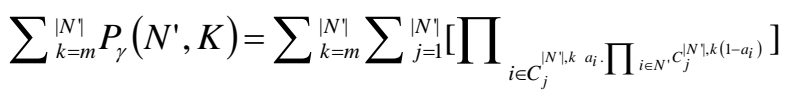

F2: Calculate Minimal price of cloud.

$\min _{j \in\left[1,\left(\left|N^{\mid}\right|, m\right)\right]}\left\{\sum_{i_{j}^{\left|N^{\top}\right|, m}} \operatorname{Sm} C_{\gamma} P_{b i}+\sum_{i_{j}^{\left|N^{\gamma}\right|, m}} C_{\gamma} P_{b i}\right\}$

F3: Synthetical price of storage.

$P i=S P_{s i}+C_{\gamma} S P_{b i}+c_{r} P_{o i}$

FO: Set of selected clouds and minimal cost Csm

q0: F1:

FP: FO: $\{$ I1; I2; I3; I4 $\}$

Where, I1: File size S, Current File Read freqCr

I2: Multiple Cloud

I3: Cloud and cloud price

I4: Size of file, Read access of storage price of i cloud, outgoing bandwidth price of $\mathrm{i}$ cloud, read access of get operation price of $\mathrm{i}$ cloud.

I5: Selected cloud with minimal price and high availability 


\subsection{Algorithm}

4.3.1 Algorithm 1: Heuristic algorithm of data placement

Input: File S, read frequency, n's upper limit

Output: Selection of cloud with minimum price

1. Initialize Min cost $=\infty$ and set of cloud $=\{\}$ and arrange cloud from high to low

2. For $\mathrm{n}=2$ to $\in$

3. Set first cloud as Gs from the list of cloud Ls.

- Remove that cloud from list Ls.

- Store remaining cloud in Gc.

- Remove that cloud from list Ls

- Store remaining cloud in Gc.

4. Traverse $m=1$ to $n$ to select appropriate cloud with minimum cost for storage.

5. Calculate availability of selected cloud Gs.

if Acur $>=$ require availability then calculate minimal cost.

$\left.\sum_{k=m}^{|V|} \operatorname{P\gamma }(N, K)=\sum_{k=m}^{|N|} \sum_{j=1}^{\left|N_{k}\right|} \Pi_{i \in C_{j}^{|N|^{a i}}}\right]$

6. If Ccur is < than the Csm then assign Ccur to Csm and put the selected cloud in the list

7. If the availability does not meet the required value Exchange the cloud in the current set Gs using greedy method.

8. Firstly sort Gs by ai and Gs by Pi from low to high where $P_{i}=S P_{s i}+C_{\gamma} S P_{o i}+C \gamma P_{o i}$

9. Then exchange the cloud in Gs from the lowest ai one by one with the cloud which has lowest Pi in Gc but higher availability than that cloud in Gs. Until the availability meets the required value.

10. If the cost of obtain Gs is lower then, update Csm and $\psi$

\subsubsection{Algorithm 2: Double-Quality-Guaranteed} (DQG) Scheme

1. A multi-server framework having $m$ servers is running and waiting for the events as takes after:

2. Initialized Q queue as empty

3. Event A service request arrives at multi-server system.

4. Search for any server is available to process the request.

5. If server is available then assign the incoming request to that server,

6. Else put that request at the end of Q queue and record its waiting time

7. End If, End Event

8. When Event A server become idle.

9. Check whether Q queue is empty or not

10. If $\mathrm{Q}$ is empty then wait for new service request to arrive.
11. Else take the first request from $\mathrm{Q}$ queue and assign it to the idle server.

12. End If, End Event

13. Event: The deadline of service request is achieved

14. Rent a (temp_srv) temporary server to execute the service request and released the temp_srv when the job is done.

15. End

\subsection{Experimental Setup}

The system is developed on Java framework (version jdk 1.8) as well as the Netbeans (version 1.8) is utilized as a development tool on Windows platform. The system runs on any common machine and it does not need any specific hardware to run.

\section{RESULTS AND DISCUSSION}

The fig. 2 shows the comparison between existing and proposed system in terms of profit. The Y-axis shows profit of systems in percentage and $\mathrm{X}$-axis shows the existing and proposed algorithm. The proposed system gains profit because of appropriate selection of cloud.

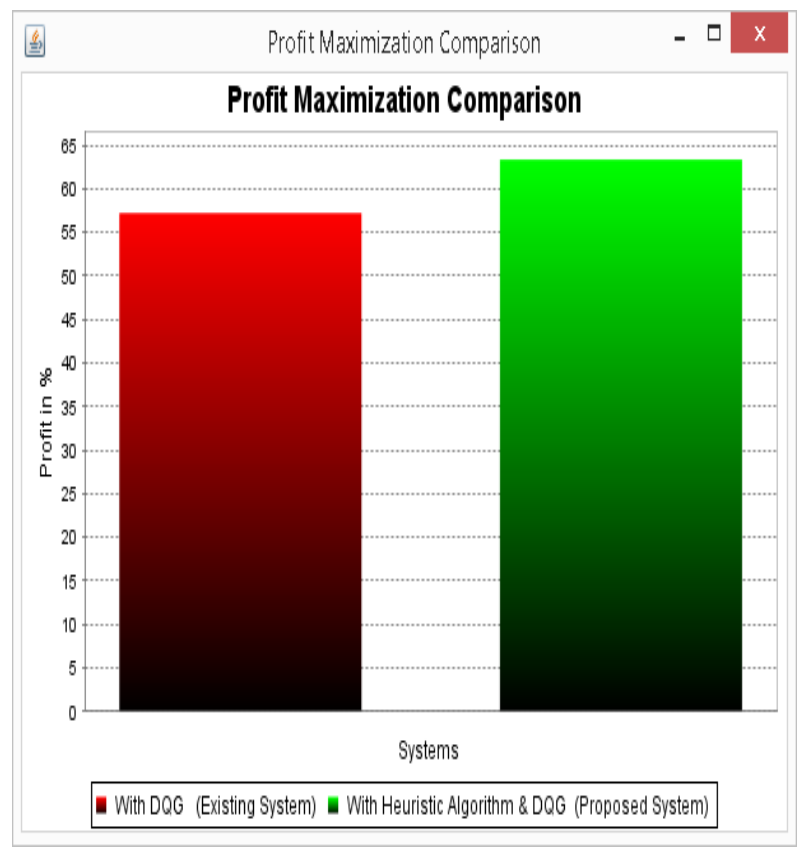

Fig.2: Profit Comparison Graph.

Table 1: Profit Maximization Comparison

\begin{tabular}{|c|c|c|}
\hline & $\begin{array}{c}\text { Existing System } \\
\text { with DQG }\end{array}$ & $\begin{array}{c}\text { Proposed System } \\
\text { with Heuristic } \\
\text { Algorithm and } \\
\text { DQG }\end{array}$ \\
\hline Profit in \% & 58 & 63 \\
\hline
\end{tabular}

The fig. 3 graph shows comparison between Optimal Servers (6 Number of Servers \& 8 Number of Servers); in this graph optimal size and optimal speed used in combination to gain the profit. In 6 numbers of servers there are more number of long term rented server, while 8 number of server contains more number of short term rented server. 
Table 2: Optimal Server Comparison

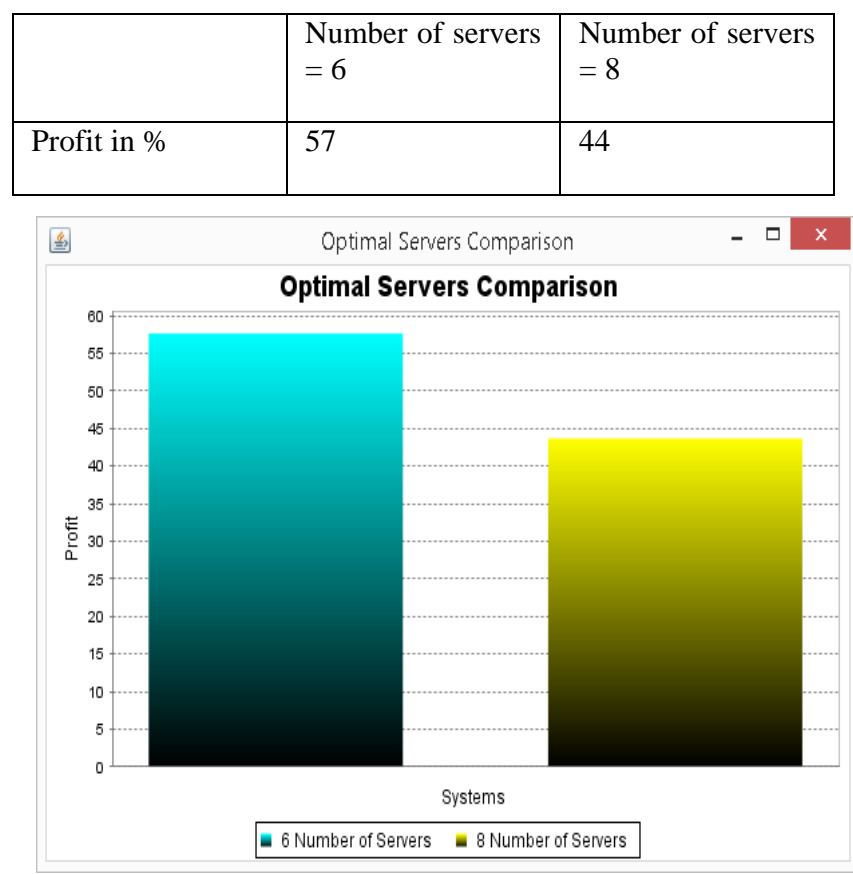

Fig3.Optimal Server Profit Comparison Graph

\section{CONCLUSION AND FUTURE SCOPE}

A new approach called Double Quality Guaranteed renting method for service providers is proposed to ensure the quality of service demands as well as to enhance the profit. To minimize the wastage of resources, this method includes both the short term renting method as well as long term renting method. To vary the system sizes, a queuing model is implemented for multi-server system. The data hosting algorithm is implemented before the DQG method, to make the suitable choice of cloud as well as to enhance the profit of service provider. After that, an issue of optimal configuration of profit increasing is defined in that several aspects are determined. This proposed double renting strategy is implemented to heterogeneous cloud environment.

\section{REFERENCES}

[1] Jing Mei, Kenli Li, Member, Aijia Ouyang and Keqin Li, Fellow, 2015 , A Profit Maximization Scheme with Guaranteed Quality of Service in Cloud Computing

[2] G. P. Cachon and P. Feldman, 2010. Dynamic versus static pricing in the presence of strategic consumers.

[3] J. Cao, K. Hwang, K. Li, and A. Y. Zomaya, , 2013. Optimal multi-server configuration for profit maximization in cloud computing.

[4] A. Fox, R. Griffith, A. Joseph, R. Katz, A. Konwinski,G. Lee, D. Patterson, A. Rabkin, and I. Stoica, 2009. Above the clouds: A berkeley view of cloud computing.

[5] R. Buyya, C. S. Yeo, S. Venugopal, J. Broberg, and I.Brandic, 2009. Cloud computing and emerging it platforms:Vision, hype, and reality for delivering computing as the5th utility.

[6] P. Mell and T. Grance, 2009. The NIST definition of cloud computing.National institute of standards and technology Information Technology Laboratory.

[7] J. Chen, C. Wang, B. B. Zhou, L. Sun, Y. C. Lee, andA. Y. Zomaya, 2011.Tradeoffs between profit and customer satisfaction for service provisioning in the cloud.

[8] J. Mei, K. Li, J. Hu, S. Yin, and E. H.-M. Sha, 2013. Energy aware preemptive scheduling algorithm for sporadic tasks on dvs platform.

[9] P. de Langen and B. Juurlink, 2009.Leakage-aware multi processor scheduling.

[10] S. Liu, S. Ren, G. Quan, M. Zhao, and S. Ren, 2013. Profit aware load balancing for distributed cloud data centers.

[11] Z. Liu, S. Wang, Q. Sun, H. Zou, and F. Yang, , 2013. Cost-aware cloud service request scheduling for saas providers. 